\title{
Bestimmung der I7-Hydroxycorticosteroide im Harn unter Verwendung von Natriummetaperjodat
}

\author{
Von P. Doerr und D. Stamm \\ Aus dem Max-Planck-Institut für Psycbiatrie, Müncben
}

(Eingegangen am 31. Dezember 1969)

Es werden die Zuverlässigkeitskriterien einer Methode zur Bestimmung der 17-OHCS') im Urin untersucht. Die Methode beruht auf der Uberführung der $\mathrm{C}_{21}$-Metabolite des Cortisols in 17-Oxosteroide durch Reduktion mit Borhydrid und anschließender Oxydation mit Natriummetaperjodat. Bei der Prüfung der Spezifität werden die gebildeten 17-Oxosteroide nach Abtrennung mit Hilfe der Girard-TReaktion dünnschichtchromatographisch und gaschromatographisch identifiziert. Die Teilschritte der Methode werden diskutiert. Die im 24 Stdn.-Urin ausgeschiedene Menge an 17-OHCS wird an 52 klinisch gesunden erwachsenen Männern und an 47 Frauen ermittelt.

\section{The determination of urinary 17-bydroxycorticosteroids in urine with sodium metaperiodate}

The reliability of a method for the determination of $17-$ OHCS in urine was investigated. The method is based on the conversion of the $\mathrm{C}_{21}$-metabolites of cortisol into 17-oxosteroids by reduction with borohydritc, followed by oxidation with sodium metaperiodate. To test the specificity, the synthesised 17-oxosteroids were separated with the aid of the Girard-T reaction and identified by thin layer and gas chromatography. Each stage of the procedure is discussed. The levels of 17-OHCS in 24 hr. urine samples are reported for 52 healthy adult men and 47 women.

Die von Appleby, Gibson, Norymberski und Stubbs (1) ausgearbeitete Methode zur Gruppenbestimmung der Cortisolmetabolite ${ }^{2}$ ) im Harn hatte sich in der klinischen Praxis allgemein durchgesetzt und bewährt. In den letzten Jahren wurden jedoch in zunehmendem Maße Methoden empfohlen (2-8), die sich im wesentlichen von der genannten Methode dadurch unterscheiden, dảß die Oxydation mit Perjodat an Stelle von Bismutat durchgeführt wird und $\mathrm{da}$ auf eine heiße Säurehydrolyse verzichtet wird. Fast alle diese Methoden basieren auf der Arbeit von FEw (9) und unterscheiden sich nur geringfügig voneinander. Der Vorteil von Natriummetaperjodat gegenüber Bismutat liegt nach FEw in der größeren Selektivität der Oxydation. Nach Reduktion der 11-, 17- und 20-Oxogruppen von Steroiden der Androstan- und Pregnanreihe mit Borhydrid wird durch Natriummetaperjodat die 17-, 20-Diol- und 17-, 20-, 21-Triolseitenkette der $C_{21}$-Steroide abgespalten. Dabei entstehen die entsprechenden 17-Oxosteroide, ohne daß die 11-Hydroxygruppe teilweise wieder oxydiert wird, wie dies bei Verwendung von Bismutat der Fall ist. Die 17-Hydroxygruppe der ursprünglich im Urin vorhandenen 17-Oxosteroide bleibt in jedem Fall reduziert. Außerdem erübrigt sich bei Gebrauch

1) $17-\mathrm{OHCS}=17-\mathrm{Hydroxycorticosteroide;} \mathrm{17-KS} \mathrm{=17-Oxoste-}$ roide; DHEA = Dehydroepiandrosteron; THE $=$ Tetrahydrocortison; $\mathrm{THF}=$ Tetrahydrocortisol; allo-THF $=$ Allotetrahydrocortisol.

2) Um möglichen Mißverständnisșen vorzubeugen sei darauf hingewiesen, daß in der vorliegenden Arbeit der Begriff 17-Hydroxycorticosteroide (17-OHCS) im Sinne von NorYMBERsKr und Mitarbeitern verwendet wird. Im englischen Sprachbereich werden die Hydroxycorticosteroide, die nach vorangegangener Reduktion der endogenen 17-Oxosteroide als 17-Oxosteroide nachgewiesen werden, als ,ketogene Steroidec bezeichnet (C. H. GRAY, D. N. BARON, R. V. Brooks und V. H. T. JAMES). von Natriummetaperjodat eine heiße Säurehydrolyse, da die Glucuronsäurekonjugate oxydativ gespalten werden. Neben dem freien Steroid soll noch der entsprechende Formiatester entstehen, der leicht alkalisch verseift werden kann. Die bei der heißen Säurehydrolyse entstehenden Artefakte werden daher vermieden. Treffen die genannten Voraussetzungen zu, so ist zu erwarten, daß sämtliche Cortisolmetabolite der 5 $\beta$-Pregnanreihe in $11 \beta$-Hydroxyätiocholanolon, die der $5 \alpha$-Pregnanreihe in $11 \beta$-Hydroxyandrosteron überführt werden.

Zweck dieser Arbeit ist es nicht, den bereits gemachten Vorschlägen zur Bestimmung der 17-OHCS noch eine weitere, eigene Modifikation hinzuzufügen; vielmehr sollen die Zuverlässigkeitskriterien, insbesondere die Spezifität, die von den meisten Autoren nicht untersucht wurde, geprüft werden. Unter den gemachten Vorschlägen, die Methode von FEw den Bedürfnissen der klinischen Routine anzupassen, entspricht die hier beschriebene Methode am meisten dem Vorschlag von WIISON und LIPSETr (2). Auf die einzelnen Teilschritte soll nur dann eingegangen werden, wenn sich unsere Ergebnisse von den in der Literatur mitgeteilten unterscheiden.

\section{Methodik}

\section{Reagenzien und Geräte}

Soweit nicht anders vermerkt, werden Reagenzien der Fa. Merck A. G., Darmstadt, vom Reinheitsgrad p. a. benutzt.

Zur Routinebestimmung der 17-OHCS werden benötigt:

Reagenzien: Diäthyläther (peroxydfrei), Chloroform, Athanol, absol. rein (Reinigung: 1 l A thanol wird mit $4 \mathrm{~g}$ m-Phenylendiammoniumdichlorid versetzt, 10-14 Tage bei gelegentlichem Umschüteln im Dunkeln aufbewahrt und dann destilliert), Essigsäure 96proz., Natriumhydroxidplätzchen, Kaliumhydroxidplätzchen, Natriumborhydrid, Natriummetaperjodat, Kaliumhydrogenphosphat, Natriumsulfat (wasserfrei), 1,3-Dinitrobenzol 
zur 17-Oxostcroid-Bestimmung, Dehydroepiandrosteron $(3 \beta-$ Hydroxy-5-androsten-17-on).

Lösungen: Natronlauge $0,1 \mathrm{~N}, 2,5 \mathrm{~N}$ und $5 \mathrm{~N}$, Kalilauge $5 \mathrm{~N}$ (titrimetrisch einstellen), Phosphatpuffer pH $71 \mathrm{M}$, Essigsäurc 25proz., Natriumborhydrid 10proz. in $0,1 \mathrm{~N} \mathrm{NaOH}$ (für jede Bestimmung frisch ansetzen), Natriummetaperjodat 10proz. (für jede Bestimmung frisch ansetzen), 1,3-Dinitrobenzol 2proz. in absol. Äthanol, DHEA-Stammlösung $1 \mathrm{mg} / \mathrm{ml}$ Äthanol, DHEA-Gebrauchsstandard $50 \mu \mathrm{g} / \mathrm{m} l$ Äthanol.

Geräte: Spektralphotometer PMQ II ${ }^{3}$, Rotationsverdampfer, Schüttelmaschine mit rotierender Achse, an der bis zu 24 Spitzreagenzgläser mit NS 29-Schliff, Fassungsvermögen etwa $110 \mathrm{ml}$, befestigt werden.

Zur Bestimmung der Zuverlässigkeitskriterien werden zusätzlich benötigt:

Åthanol (absolut), Äthylacetat, Benzol, Cyclohexan, Dichlormethan, Essigsäure 99-100proz., Toluol, Salzsäure (rauchend), Kaliumcarbonat, Girards Reagenz T (Trimethylacet-hydrazidammoniumchlorid), Kieselgel G ohne Fluoreszenzindikator, Träger und stationäre Phasen für die Gaschromatographie ${ }^{4}$ ): Aeropak 30, 100/120 mesh, SE-30, OV-17, QF-1; Nachweisreagenzien für die Dünnschichtchromatographie: ZimmermannReagenz: a) 2proz. 1,3-Dinitrobenzollösung in Äthanol, b) 2,5N methanolische $\mathrm{KOH}$; die Lösungen a) und b) werden direkt vor Gebrauch gemischt; Anisaldehyd-Schwefelsäure-Reagenz nach LISBOA (10).

Steroide: Androsteron ( $3 \alpha$-Hydroxy-5 $\alpha$-androstan-17-on), Epiandrosteron ( $3 \beta$-Hydroxy-5 $\alpha$-androstan-17-on), Tetrahydrocortison (3a,17 $\alpha, 21$-Trihydroxy-5 $\beta$-pregnan-11, 20-dion), Tetrahydrocortisol ( $3 \alpha, 11 \beta, 17 \alpha, 21$-Tetrahydroxy-5 $\beta$-pregnan-20-on), Pregnantriol $(3 \alpha, 17 \alpha, 20 \alpha$-Trihydroxy-5 $\beta$-pregnan), Cholesterin ( $3 \beta$-Hydroxy-cholest-5-en); die folgenden Steroide werden von der Fa. Ikapharm ${ }^{5}$ ) bezogen: Ätiocholanolon ( $3 \alpha-H y d r o x y-5 \beta-$ androstan-17-on), 11 $\beta$-Hydroxyäthiocholanolon $(3 \alpha, 11 \beta$-Dihydroxy- $5 \beta$-androstan-17-on), $11 \beta$-Hydroxyandrosteron $(3 \alpha, 11 \beta$ Dihydroxy-5 $\alpha$-androstan-17-on), 11-Oxoätiocholanolon ( $3 \alpha$-Hydroxy-5 $\beta$-androstan-11, 17-dion), 11-Oxoandrosteron (3 $\alpha$-Hydroxy-5 $\alpha$-androstan-11, 17 -idon), Allotetrahydrocortisol $(3 \alpha, 11 \beta$, $17 \alpha, 21$-Tetrahydroxy-5 $\alpha$-pregnan-20-on).

Bei der Dünnschichtchromatographie werden $200 \times 200 \mathrm{~mm}$ Glasplatten benutzt. Die Beschichtung erfolgt mit dem automatischen Beschichtungsgerät 216026).

Zur Gaschromatographie wird das Modell 2120 der Fa. Varian MAT mit zwei Flammenionisationsdetektoren benutzt.

\section{Durchfiibrung der Routinebestimmung der 17-OHCS}

$10 \mathrm{~m} l$ Urin werden in ein Spitzreagenzglas mit Schliff (Fassungsvermögen etwa $110 \mathrm{ml}$ ) pipettiert und auf $\mathrm{pH} 7 \mathrm{mit} 0,1 \mathrm{~N} \mathrm{NaOH}$ (Kontrolle mit pH-Papier, Bereich 6-8) eingestellt. Anschließend wird $1 \mathrm{~m} l$ der Natriumborhydridlösung zugegeben. Mit etwa $1 \mathrm{~m} /$ Diäthyläther kann übermäßige Schaumbildung leicht vermieden werden. Nach einer Stunde Reduktion bei Raumtemperatur wird Natriumborhydrid durch Ansäuern mit $0,8 \mathrm{~m} /$ 25proz. Essigsäure zerstört. Nach einer weiteren Viertelstunde pipettiert man in jedes Glas $4 \mathrm{ml}$ der Natriummetaperjodatlösung und stellt das Reaktionsgemisch mit $2,5 \mathrm{~N} \mathrm{NaOH}$ auf einen $\mathrm{pH}$ Wert zwischen 6,5 und 7,0 ein. Die Reaktionsgefäße werden in ein Wasserbad von $37^{\circ}$ gestellt. Nach Ablauf einer Stunde wird das Reaktionsgemisch mit $0,5 \mathrm{ml} 5 \mathrm{~N} \mathrm{NaOH}$ alkalisch gemacht. Nach weiteren $20 \mathrm{Min}$. werden die Spitzreagenzgläser aus dem Wasserbad genommen, unter Leitungswasser abgekühlt und die gebildeten 17-Oxosteroide mit $80 \mathrm{ml}$ Äther unter Verwendung der Schüttelmaschine (etwa $60 \mathrm{U}$./Min.) ausgeschüttelt. Die Ätherphase wird jeweils mit $5 \mathrm{~m} l 2,5 \mathrm{~N} \mathrm{NaOH}, 5 \mathrm{ml}$ Phosphatpuffer und $3 \mathrm{~m} l$ demin. Wasser gewaschen. Die Unterphase wird mit Hilfe

3) Fa. C. Zeiss, Oberkochen/Württ.

4) Fa. Varian MAT, Walnut Creek, Calif., USA.

5) Fa. Ikapharm, Ramat-Gan, Israel.

6) Fa. Camag, Muttenz, Schweiz. einer $20 \mathrm{~m}$-Rekordspritze mit aufgesetzter Punktionsnadel abgesaugt. Die mit Natriumsulfat getrocknete Ȧtherphase wird quantitativ in einen $150 \mathrm{ml}$-Birnenkolben überfühtt und am Rotationsverdampfer im Wasserbad von $45^{\circ}$ abgedampft. Bei Verwendung einer Destillationsspinne können 4 Birnenkolben gleichzeitig abgedampft werden. Analog den Urinproben werden als Leerwert $10 \mathrm{~m} l$ Wasser durch den gesamten Analysengang geführt. Es genügen insgesamt 3 Leerwertansätze.

Nach Abdampfen des Äthers wird der Rückstand der Proben und Leerwerte in $2,5 \mathrm{~m} l$ des gereinigten Alkohols aufgenommen. $1 \mathrm{ml}$ Lösung wird zur Zimmermann-Reaktion benötigt. Normalerweise wird eine Doppelbestimmung durchgeführt.

\section{Zimmermann-Reaktion}

In Schliffreagenzgläser (NS 14,5,15-20 $\mathrm{m} l$ Fassungsvermögen) werden pipettiert: $1 \mathrm{ml}$ des in Äthanol aufgenommenen Rückstandes der Proben (P), $1 \mathrm{~m} l$ des Leerwertes (TRL), $1 \mathrm{~m} l$ des DHEA-Gebrauchsstandards (St), $1 \mathrm{ml}$ des gereinigten Äthanol als Reagenzienleerwert der Farbreaktion .(RL). In jedes Reagenzglas werden zusätzlich pipettiert: $1 \mathrm{~m} l$ Äthanol, $2 \mathrm{ml}$ der 1,3-Dinitrobenzollösung und $2 \mathrm{ml} 5 \mathrm{~N} \mathrm{KOH}$. Der Reaktionsansatz bleibt 90 Min. im abgedunkelten Wasserbad bei $25^{\circ}$. Anschließend gibt man $2 \mathrm{ml}$ demin. Wasser und $5 \mathrm{ml}$ Chloroform hinzu, schüttelt 10-15 Sek. und läßt etwa 5-10 Min. zur Phasentrennung stehen. Die gelblichbraune Oberphase wird abgesaugt und verworfen, die Chloroformphase in ein Zentrifugenglas filtriert. Die Extinktionen (E) der Proben (P), der Leerwerte (TRL, RL) und des Standards (St) werden bei $510 \mathrm{~nm}$ gegen Chloroform in $1 \mathrm{~cm}$-Schichtküvetten gemessen.

\section{Berechnung}

$$
\begin{aligned}
& \frac{\left(\mathrm{E}_{\mathrm{P}}-\mathrm{E}_{\mathrm{TRL}}\right) \cdot 50 \cdot 1,34 \cdot 1,19 \cdot 2,5 \cdot \text { Harnmenge in } l}{\left(\mathrm{E}_{\mathrm{St}}-\mathrm{E}_{\mathrm{RL}}\right) \cdot 10} \\
&= \frac{\left(\mathrm{E}_{\mathrm{P}}-\mathrm{E}_{\mathrm{TRL}}\right) \cdot 19,9 \cdot \text { Harnmenge in } l}{\mathrm{E}_{\mathrm{St}}-\mathrm{E}_{\mathrm{RL}}} \quad \mathrm{mg} \mathrm{17-OHCS \text {in }} \\
& \text { THE-Äquivalenten }
\end{aligned}
$$

\section{Zur Berechnung:}

1,34: Faktor zur Umrechnung des prozentualen Extinktionskoeffizienten von $11 \beta$-Hydrōxyätiocholanolon (Hauptbestandteil der Probe) in den des Standards

1,19: Faktor zur Umrechnung von mg DHEA in mg THE.

\section{Priifung der Spezifität}

Zur Untersuchung der Spezifität der beschriebenen Methode werden die alkoholischen Steroide mit Hilfe von Girards Reagenz $T$ aus dem sogenannten Harnextrakt (Rückstand nach Abdampfen der Ätherphase) abgetrennt, bevor die gebildeten 17-Oxiosteroide dünnschichtchromatographisch und gaschromatographisch identifiziert werden. Abweichend von der Vorschrift von PINcus und Pearlman (11) wird das Reaktionsgemisch mit einer Kaliumcarbonatlösung und Phosphatpuffer innerhalb weniger Sekunden neuttalisiert, so daß ein rasches Arbeiten bei niedrigen Temperaturen nicht nötig ist.

Girard-T-Trennung: Der in ein Spitzreagenzglas überführte neutrale Harnextrakt wird in $0,5 \mathrm{~m} l$ Eisessig gelöst und mit etwa $50 \mathrm{~m} l$ Girards Reagenz T versetzt. Nach Erhitzung des Reaktionsgemisches im Glycerinbad auf $100^{\circ}$ für $25 \mathrm{Min}$. werden die Gefäße im kalten Leitungswasser abgekühlt und das Reaktionsgemisch mit $3 \mathrm{~m} / 1,2 \mathrm{~N} \mathrm{~K}_{2} \mathrm{CO}_{3}$ und mit $5 \mathrm{ml}$ 0,5M Phosphatpuffer, $\mathrm{pH} 7$, neutralisiert. Die alkoholischen Steroide werden mit $80 \mathrm{ml}$ Äther ausgeschüttelt und verworfen. Die Hydrazone der 17Oxosteroide werden nach vorsichtiger Zugabe von $3 \mathrm{~m} l$ rauchender Salzsäure im Wasserbad bei $37^{\circ}$ in eineinhalb Stunden wieder hydrolysiert. Anschließend Ausschütteln mit $80 \mathrm{ml}$ Äther oder $40 \mathrm{ml}$ Dichlormethan, Waschen der organischen Phase mit $3 \mathrm{ml}$ $2,5 \mathrm{~N} \mathrm{NaOH}$ zur Entfernung der Essigsäure und Waschen mit demin. Wasser bis zur Neutralität. Zur Durchführung der Dünnschichtchromatographie oder Gaschromatographie wird der Rückstand in ein geeignetes Gefäß überführt und in $40 \mu l$ Toluol aufgenommen. 
Dunnscbicbtcbromatograpbie: Zur Trennung der $\mathrm{C}_{18} \mathrm{O}_{2}$-17-Oxosteroide wird das System Cyclohexan/Äthylacetat 50:50 (v/v), zur Trennung der $\mathrm{C}_{19} \mathrm{O}_{3}-17-$-Oxosteroide das System Chloroform/ Athanol 95:5 (v/v) benutzt. Beide Systeme wurden in gleicher oder nur wenig abweichender Zusammensetzung von LisBOA (10) zur Trennung von Steroiden angewandt und sind dort ausführlich diskutiert.

Gascluromatographie: Die Gaschromatographie wird als weiteres Verfahren zur Trennung und Identifizierung der 17-Oxosteroide des neutralen Harnextrakts benutzt. Die $\mathrm{C}_{19} \mathrm{O}_{2^{-}}$und $\mathrm{C}_{19} \mathrm{O}_{3}-17-$ Oxosteroide werden sowohl als freie Steroide wie auch als Trimethylsilyläther chromatographiert. Sollen $\mathrm{C}_{18} \mathrm{O}_{2}$ - und $\mathrm{C}_{18} \mathrm{O}_{3}-17-$ Oxosteroide zugleich getrennt werden, erfolgt die Chromatographie der Verbindungen als freie Steroide auf OV-17.

\section{Ergebnisse und Diskussion}

Untersuchung der einzelnen Teilschritte der Methode

\section{Redultion der Oxogruppen}

An reinen Lösungen $(10 \mathrm{~m} /$ Wasser +100 oder $200 \mu \mathrm{g}$ THE) war die Reduktion der 11-Oxo-Gruppe im Gegensatz zum Urin bei den Bedingungen der angegebenen Methode unvollständig. Die dünnschichtchromatographische und gaschromatographische Auftrennung des Rückstandes der eingedampften Ätherphase zeigte neben 11 $\beta$-Hydroxyätiocholanolon noch eine weitere Substanz mit gleichem Verhalten wie 11-Oxoätiocholanolon, die etwa 30-50\% der Gesamtmenge ausmachte. Wurde die Reduktionszeit oder die Borhydridkonzentration verdoppelt oder die Temperatur des Reaktionsgemisches auf $37^{\circ}$ erhöht, so fand sich nur noch $11 \beta$ Hydroxyätiocholanolon. $\mathrm{Da}$ es sich um eine unvollständige Reduktion und nicht um eine nachträgliche Oxydation mit Natriummetaperjodat handelt, folgt aus den entsprechend durchgeführten Versuchen mit THF. Hier wurde bei sämtlichen Reduktionsbedingungen nur $11 \beta$-Hydroxyätiocholanolon gefunden.

Die Versuche, bei denen $200 \mu \mathrm{g}$ THE zu $10 \mathrm{ml}$ Urin aufgestockt wurden, zeigten bei der gaschromatographischen Trennung des Rückstandes nach der GirardT-Trennung nur einen recht kleinen, aber noch über der Nachweisgrenze gelegenen Peak mit der gleichen relativen Retentionszeit wie 11-Oxoätiocholanolon. Wurde die Reduktionszeit oder die Konzentration an Borhydrid verdoppelt, so verschwand dieser Peak. Von praktischer Bedeutung scheint der offenbar sehr geringe, nicht reduzierte Anteil der 11-Oxo-Gruppe nicht zu sein: 1. Eine Stichprobe von 10 Sammelurinen, deren Gehalt an 17-OHCS an der oberen Grenze unseres Normalkollektivs lag, wurde zusätzlich unter Verwendung der doppelten Borhydridkonzentration analysiert. Die Differenz der arithmetischen Mittel der unterschiedlich aufgearbeiteten Proben betrug $2 \%$ und war statistisch nicht signifikant (Signed-Rank-Test nach WILKoxON). 2. Auch die Tatsache, daß die Wiederauffindungstaten von THE und THF im Urin (s. Tab. 2) sich im Rahmen der durch Zufallsfehler bedingten Vertrauensgrenzen nicht unterscheiden, spricht dafür, daß die Reduktionsbedingung der angegebenen Methode ausreichend ist.
Die 17-Oxo-Gruppen wurden vollständig reduziert. Die entstandenen 17-Hydroxy-Gruppen wurden durch $\mathrm{Na}$ triummetaperjodat unter den angegebenen Bedingungen nicht wieder oxydiert. 10 Urinproben wurden teils mit $100 \mu \mathrm{g}$ Ätiocholanolon, teils mit $100 \mu \mathrm{g} 11 \beta$-Hydroxyätiocholanolon aufgestockt. In den nicht aufgestockten und den aufgestockten Proben wurden gleich viel 17-OHCS gemessen.

\section{Oxydation}

Hinsichtlich der Reaktionsbedingungen bei der $\mathrm{Na}$ triummetaperjodatoxydation stimmten unsere Ergebnisse mit den in der Literatur $(2,4,9)$ mitgeteilten überein.

\section{Wabl des organiscben Lösungsnittels zum Ausscbiitteln der gebildeten 17-Oxostoroide}

Äther hat den Vorteil, daß beim Ausschütteln von Urin keine Emulsionen auftreten. Zusätzlich läßt sich die zu verwerfende Unterphase in einem großen Spitzenreagenzglas gut absaugen. Der Verteilungskoeffizient der $\mathrm{C}_{19} \mathrm{O}_{3}-17-\mathrm{Oxosteroide}$ ist jedoch bei anderen Lösungsmitteln günstiger als bei Äther. So wurden nach Einstellen des Verteilungsgleichgewichts zwischen gleich großen Volumina wäßriger und organischer Phase $99-100 \%$ von $11 \beta$-Hydroxyätiocholanolon in der Dichlormethanphase und nur $90 \%$ in der Ätherphase gefunden.

\section{Zuverlässigkeitskriterien}

\section{Präzision in der Serie und von Tag z" Tag}

Aus dem frisch gesammelten Urin mehrerer klinisch gesunder, medikamentfreier Individuen wurden nach gründlichem Durchmischen 200 Proben von etwa $25 \mathrm{ml}$ in kleine Kautexflaschen abgefüllt und in der Tiefkühltruhe bei $-18^{\circ}$ eingefroren. Mit jeder Analysenserie wurde zunächst eine Doppelbestimmung und später nur noch eine Einfachbestimmung durchgeführt. Das Ergebnis der Berechnung der Präzision in der Serie aus 24 Doppelbestimmungen und der Präzision von Tag zu Tag gibt die Tabelle 1 wieder.

Tab. 1

Präzision der Methode

\begin{tabular}{|c|c|c|c|}
\hline & & $\begin{array}{l}\text { Präzision } \\
\text { in der Serie }\end{array}$ & $\begin{array}{c}\text { Präzision } \\
\text { von Tag zu Tag }\end{array}$ \\
\hline $\begin{array}{l}\text { arithm. Mittel } \\
\text { Anzahl }\end{array}$ & $\begin{array}{l}\bar{x} \\
\mathrm{n}\end{array}$ & $\begin{array}{c}12,5 \mathrm{mg} / \mathrm{l} 17-\mathrm{OHCS} \\
24 \text { Doppel- } \\
\text { bestimmungen }\end{array}$ & $\begin{array}{c}12,4 \mathrm{mg} / \mathrm{l} 17-\mathrm{OHCS} \\
34 \text { Einzel- } \\
\text { bestimmungen }\end{array}$ \\
\hline $\begin{array}{l}\text { Standardabweichung } \\
\text { Variationskoeffizient }\end{array}$ & $\stackrel{s}{\mathbf{v}}$ & $\begin{array}{l}0,28 \\
2,3 \%\end{array}$ & $\begin{array}{l}0,73 \\
5,9 \%\end{array}$ \\
\hline
\end{tabular}

\section{Nacbiveisgrenze}

Aus der Standardabweichung der Leerwertextinktionen $\left(E_{\text {TRL }}\right)$ an 24 verschiedenen Tagen wurde die Nachweisgrenze berechnet (12).

Nachweisgrenze

$$
\begin{aligned}
& =\frac{19,9 \cdot 0,0064 \cdot 3}{0,250} \\
& =1,53 \mathrm{mg} / / 17-\mathrm{OHCS} \text { als THE-Äquivalente }
\end{aligned}
$$


0,0064: Standardabweichung der Leerwertextinktionen bei $\mathrm{n}=24$ und $\overline{\mathrm{x}}=0,071$

0,250: Extinktion des DHEA-Gebrauchsstandards

19,9: Ableitung dieses Faktors siehe unter Methodenbeschreibung

\section{Wiederauffindungsraten}

100 und $200 \mu \mathrm{g}$ THE, $200 \mu \mathrm{g}$ THF, 100 und $200 \mu \mathrm{g}$ allo-THF und $116 \mu \mathrm{g}$ Pregnantriol (entsprechend $100 \mu \mathrm{g}$ Ätiocholanolon) wurden jeweils $\mathrm{zu} 10 \mathrm{ml}$ eines Poolurins in $100 \mu l$ Äthanol hinzugegeben. Aus Tabelle 2 sind die wiedergefundenen Mengen in Prozent der eingesetzten ersichtlich. Die Berechnung von THE und THF erfolgt nach der Angabe bei der Methodenbeschreibung. Im Falle von allo-THF wurden die Zimmeräquivalente von $11 \beta$-Hydroxyandrosteron berücksichtigt, die bei der benutzten Farbreaktion um 11,4\% niedriger lagen als für $11 \beta$-Hydroxyätiocholanolon. Zur Bestimmung der Wiederauffindungsrate von Pregnantriol wurde an Stelle des DHEA-Gebrauchsstandards eine alkoholische Lösung von Ätiocholanolon, $50 \mu \mathrm{g} /$ $\mathrm{ml}$, benutzt.

Tab. 2

Wiederauffindungsraten von THE, THF, allo-THF und Pregnantriol in Prozent der eingesetzten Mengen. Angegeben ist jeweils die Menge, die $10 \mathrm{ml}$ Urin zugesetzt wurde. $\Delta \overline{\mathrm{x}}$ ist der $95 \%$ Vertrauensbereich des arithmetischen Mittels

\begin{tabular}{lccc}
\hline & $100 \mu \mathrm{g} \mathrm{THE}$ & $200 \mu \mathrm{g}$ THE & $200 \mu \mathrm{g}$ THF \\
$\overline{\mathbf{x}}$ & 86,8 & 84,8 & 84,7 \\
$\Delta \overline{\mathrm{x}}$ & $\pm 5,4$ & $\pm 3,0$ & $\pm 4,2$ \\
$\mathrm{n}$ & 11 & 10 & 8 \\
\hline & $100 \mu \mathrm{g}$ allo-THF & $200 \mu \mathrm{g}$ allo-THF & $116 \mu \mathrm{g}$ Pregnantriol \\
$\overline{\mathrm{x}}$ & 85,1 & 87,5 & 82,6 \\
$\Delta \overline{\mathrm{x}}$ & $\mathbf{4}$ & $\pm 6,7$ & $\pm 1,6$ \\
$\mathrm{n}$ & & 8 & 8 \\
\hline
\end{tabular}

\section{Sperifität}

Alle Substanzen, die keine Metabolite des Cortisols sind und einen Beitrag zur Farbreaktion des neutralen Harnextraktes liefern, schränken die Spezifität der Methode ein. Sie können formal unterteilt werden in Chromogene, die nichts mit dem Steroidstoffwechsel zu tun haben, und in Steroide mit Oxogruppen, die nicht vom Cortisolstoffwechsel stammen. Falls die in der Einleitung zusammengefaßten und an anderer Stelle (9) ausführlich begründeten Voraussetzungen hinsichtlich der Reaktionsabfolge Borhydridreduktion - Perjodatoxydation zutreffen, sollten sämtliche Cortisolmetabolite der $5 \beta$ Pregnanreihe in $11 \beta$-Hydroxyätiocholanolon, die der $5 \alpha$-Pregnanreihe in $11 \beta$-Hydroxyandrosteron überführt werden. Zur Überprüfung dieser Annahme wird der neutrale Harnextrakt von 10 verschiedenen Normalpersonen sowie der neutrale Harnextrakt eines gepoolten Urins mit und ohne Aufstockzusätze von THE, THF, allo-THF und Pregnantriol hergestellt. Anschließend , wird eine Girard-T-Trennung durchgeführt, da hierbei nur Substanzen entfernt werden, die keinen Beitrag zur Zimmermann-Reaktion liefern, aber bei der dünnschichtchromatographischen und gaschromatographischen Auftrennung der $\mathrm{zu}$ erwartenden Steroide stören.
Die dünnschichtchromatographische Auftrennung des Rückstandes nach der Girard-T-Trennung zeigte bei den einzelnen Normalurinen und dem Poolurin jeweils drei Flecke, die in ihrem Verhalten mit 11 $\beta$-Hydroxyätiocholanolon, $11 \beta$-Hydroxyandrosteron und Ätiocholanolon übereinstimmten. Sie hatten in den benutzten Systemen übereinstimmende $R_{F}$-Werte mit den Referenzsubstanzen, waren zimmermannpositiv und zeigten die charakteristische Fluoreszenz dieser Substanzen nach Reaktion mit dem Anisaldehyd-Schwefelsäure-Reagenz. In der Region der $\mathrm{C}_{18} \mathrm{O}_{3}$-17-Oxosteroide konnte das Vorkommen von 11-Oxoätiocholanolon und 11-Oxoandrosteron, in der Region der $\mathrm{C}_{19} \mathrm{O}_{2}$-17-Oxosteroide das Vorkommen von Androsteron, Epiandrosteron und Dehydroepiandrosteron ausgeschlossen werden. Bei den mit THE und THF aufgestockten Urinen nahm der dem 11 $\beta$-Hydroxyätiocholanolon entsprechende Fleck an Größe und Intensität zu, analog führte allo-THF zu $11 \beta$-Hydroxyandrosteron und Pregnantriol zu Ätiocholanolon.

Die Eigenschaften des benutzten gaschromatographischen Systems und die relativen Retentionszeiten der $\mathrm{C}_{19} \mathrm{O}_{2}$-17-Oxosteroide und der $\mathrm{C}_{19} \mathrm{O}_{3}$-17-Oxosteroide sind in Tabelle 3 wiedergegeben. Das Ergebnis der gaschromatographischen Auftrennung des Poolurins (Rückstand nach Girard-T-Trennung) mit und ohne Zusätze von THE, THF, allo-THF und Pregnantriol zeigen die Abbildungen $1 \mathrm{a}-\mathrm{f}$.

Tab. 3

Relative Retentionszeiten der freien $\mathrm{C}_{10} \mathrm{O}_{2}-$ und $\mathrm{C}_{10} \mathrm{O}_{8}-17-0 x$ osteroide bei den gaschromatographischen Bedingungen: Glassäule $180 \mathrm{~cm}$ Länge, $2 \mathrm{~mm}$ innerer Durchmesser, Träger Aeropak 30, 100/120 mesh, beladen mit $1 \%$ OV-17, Arbeitstemperatur $255^{\circ}$ isotherm, Detektor und Injektor $315^{\circ}$, Trägergas Stickstoff, $20 \mathrm{ml} / \mathrm{Min}$., Wasserstoff $20 \mathrm{~m} l / M i n .$, synthetische Luft $300 \mathrm{ml} / \mathrm{Min}$., Gesamtretentionszeit von Cholesterin 14 Min. 35 Sek.

$\begin{array}{ll}\text { Ätiocholanolon } & 0,35 \\ \text { Androsteron } & 0,38 \\ \text { Epiandrosteron } & 0,385 \\ \text { Dehydroepiandrosteron } & 0,39 \\ \text { 11-Oxoätiocholanolon } & 0,48 \\ \text { 11-Oxoandrosteron } & 0,55 \\ \text { 11 } \beta \text {-Hydroxyätiocholanolon } & 0,65 \\ \text { 11 } \beta \text {-Hydroxyandrosteron } & 0,73 \\ \text { Cholesterin } & 1,00\end{array}$

Das Chromatogramm des Poolurins zeigt drei Peaks, die in ihren relativen Retentionszeiten mit Ätiocholano= lon, $11 \beta$-Hydroxyätiocholanolon und $11 \beta$-Hydroxyandrosteron übereinstimmen. Unter Berücksichtigung der dünnschichtchromatographischen Ergebnisse kann es als gesichert gelten, daß es sich tatsächlich um diese Substanzen handelt. Zumischen von THE und THF führte $\mathrm{zu} 11 \beta$-Hydroxyätiocholanolon, allo-THF $\mathrm{zu}$ $11 \beta$-Hydroxyandrosteron und Pregnantriol zu Ätiocholanolon. Auch die übrigen analysierten Normalurine. zeigten die gleichen Peaks, wobei erwartungsgemäß $11 \beta$-Hydroxyätiocholanolon immer die Hauptkomponente darstellte.

Es wurden keine Aufstockversuche mit Cortolen und Cortolonen durchgeführt, da THE, THF und allo-THF nur dann in $11 \beta$-Hydroxyätiocholanolon bzw. $11 \beta$ Hydroxyandrosteron überführt werden, wenn sie zuvor 
zu den entsprechenden Cortolen reduziert werden. Es konnte somit gezeigt werden, daß bei der beschriebenen Methode die Cortisolmetabolite der 5 $\beta$-Pregnanreihe in $11 \beta$-Hydroxyätiocholanolon, die der $5 \alpha$-Pregnanreihe in $11 \beta$-Hydroxyandrosteron überführt werden.

Die Spezifität der Methode wird eingeschränkt durch Metabolite des $17 \alpha$-Hydroxyprogesterons (im wesentlichen Pregnantriol) und des 11-Desoxycortisols, die zu Ätiocholanolon führen. Der Anteil des Ätiocholanolons an den Zimmermannchromogenen soll bei Normalpersonen etwa $15 \%$ betragen (9), was nach unseren Chromatogrammen glaubhaft erscheint. Bei pathologischen Zuständen (Adrenogenitales Syndrom) kann dieser Anteil wesentlich größer sein. Hier würde die chromatographische Auftrennung des neutralen Harnextraktes eine zusätzliche Information bringen. Die Spezifität ist andererseits noch durch Metabolite des normalerweise nicht sezernierten 21-Desoxycortisols eingeschränkt, die zu denselben $\mathrm{C}_{18} \mathrm{O}_{3}$-17-Oxosteroide führen wie die Cortisolmetabolite.

Die Frage, ob die Methoden, die Natriummetaperjodat verwenden, im Vergleich zur Methode von AppLEBy, Grbson, Norymberskx und Stubbs (1) der klinischen Routinediagnostik einen Informationszuwachs bringen, möchten wir trotz ihrer nachweislich höheren Spezifität offen lassen. Immerhin bietet Natriummetaperjodat den Vorteil der guten Wasserlöslichkeit. Außerdem sind wesentliche Unterschiede $z$ wischen den p. a.-Produkten der verschiedenen Hersteller, wie dies für Bismutat der Fall ist, bisher nicht bekannt geworden. Soll der neutrale Harnextrakt zur getrennten Bestimmung von Ätiocholanolon, $11 \beta$-Hydroxyätiocholanolon und $11 \beta$-Hydroxyandrosteron weiter aufgearbeitet werden, empfieblt sich die Verwendung von Natriummetaperjodat.

\section{Normalwerte}

52 Männer im Alter von 18 bis 66 Jahren und 47 Frauen im Alter von 18 bis 62 Jahren, die klinisch gesund waren und keinerlei Medikamente zu sich nahmen, sammelten gegen eine Belohnung einen 24-Stdn.-Urin. Auf ein Konservierungsmittel wurde verzichtet, da noch weitere Bestandteile bestimmt werden sollten. Während des Sammelns wurde der Urin im Kühlschrank oder in einer Kühltasche aufbewahrt. Bei der Gewinnung der Normalwerte wurde mit jeder Analysenserie noch eine Doppelbestimmung der Proben des Urinpools durchgeführt, der bereits bei der Ermittlung der Präzision der Methode verwandt wurde. Zeitabhängige systematische Fehler (Trends) traten nicht auf, die Präzision entsprach der für die Methode ermittelten.

\section{Abb. $1 \mathrm{a}-\mathrm{f}$}

Die gaschromatographischen Bedingungen sind in Tab. 3 wiedergegeben

a) Testchromatogramm: Reihenfolge der Steroide mit zunehmenden Retentionszeiten: Atiocholanolon, Androsteron, 11-Oxoätiocholanolon, 11 -Oxoandrosteron, $11 \beta$-Hydroxyätiocholanolon, $11 \beta-H$ ydroxyandrosteron, Cholesterin. Es wurden etwa $0,5 \mu \mathrm{g}$ von jedem 17-Oxosteroid und $1,5 \mu \mathrm{g}$ Cholesterin in $1 \mu l$ injiziert

b)-f) Chromatogramme des Poolurins b) ohne Aufstockung und mit Zusatz von c) THF, d) THE, e) allo-THF, f) Pregnantriol. Die Chromatographie erfolgte nach der Girard-T-Trennung; Cholesterin diente als interner Standard.

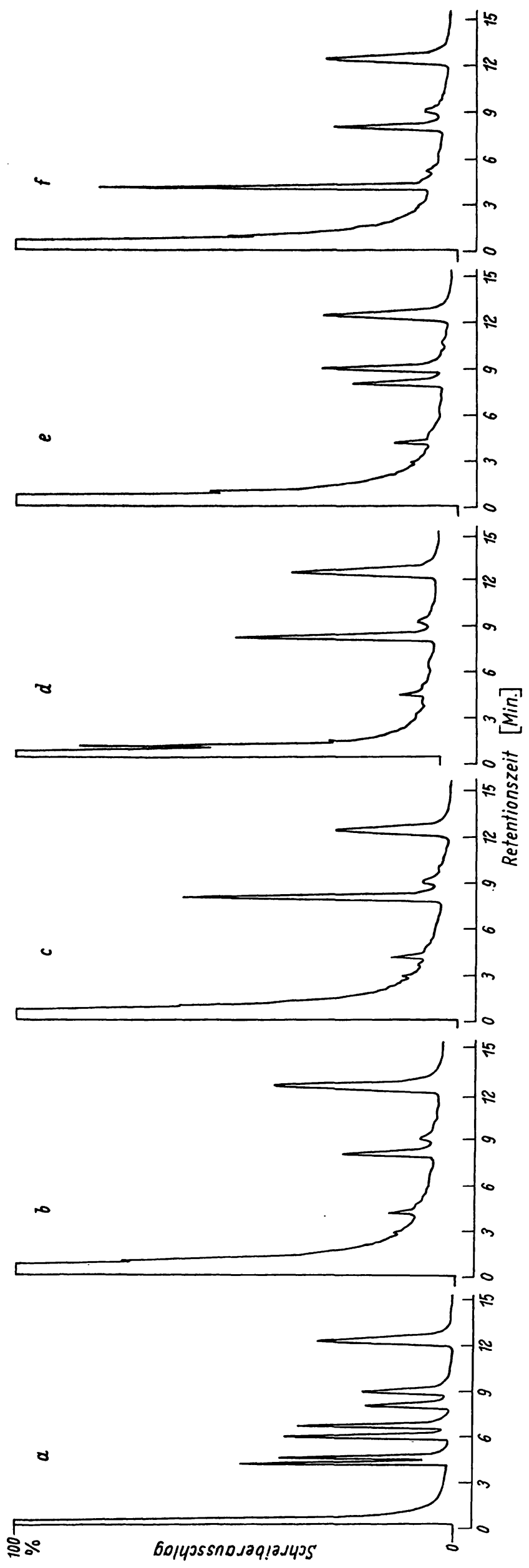

Z. klin. Chem. u. klin. Biochem. / 8. Jahrg. 1970/ Heft 3. 
Die Abbildung 2 gibt die Ausscheidung der 17-OHCS in $\mathrm{mg}$ THE-Äquivalenten je $24 \mathrm{Stdn}$. der einzelnen

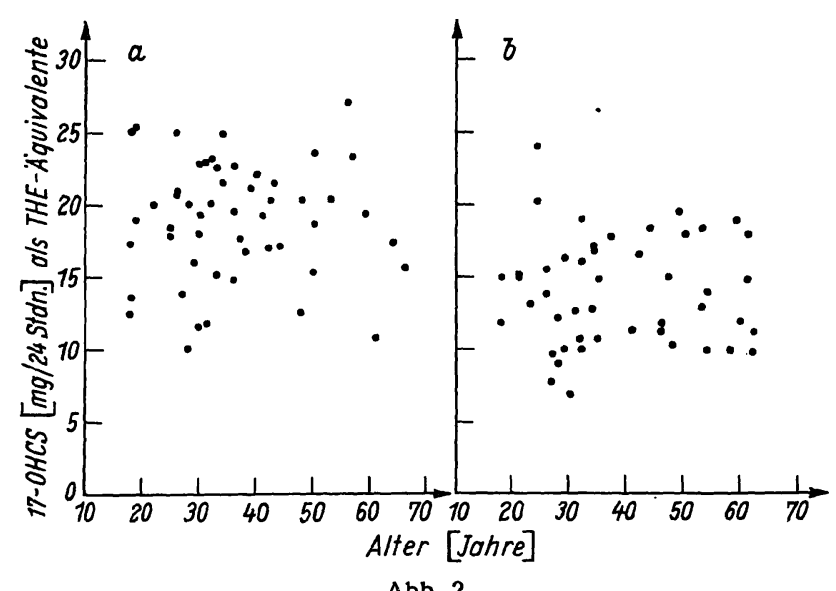

Ausscheidung der 17-OHCS in mg/24 Stdn. als THE-Äquivalente

a) $52 \mathrm{klinisch}$ gesunde Männer
b) $47 \mathrm{klinisch}$ gesunde Frauen

Tab. 4

Ausgeschiedene Menge an 17-OHCS in $24 \mathrm{Stdn}$. bei zwei Normalkollektiven. Angabe in $\mathrm{mg}$ THE-Äquivalenten, in ( ) in $11 \beta$-Hydroxyätiocholanolon-Äquivalenten

\begin{tabular}{lcc}
\hline & Männer, $\mathrm{n}=52$ & Frauen, $\mathrm{n}=47$ \\
\hline Median & 19,4 & 13,9 \\
95-Perzentilgrenzen & $(16,3)$ & $(11,7)$ \\
& 10,8 u. 25,4 & 7,8 u. 19,4 \\
& $(9,1$ u. 21,3) & $(6,6$ u. 16,3) \\
\hline
\end{tabular}

Individuen beider Kollektive in Abhängigkeit des Alters wieder. Statistisch läßt sich der Geschlechtsunterschied. sichern $(p=0,01)$. Um eine Altersabhängigkeit nachweisen zu können, hätte der Umfang der Stichproben wesentlich größer sein müssen. Die Kollektive sollen durch den Median und die 95-Perzentilgrenzen charakterisiert werden (Tab. 4), da bei dem geringen Umfang der Stichproben die Entscheidung, welches theoretische Modell der empirischen Verteilung zugrunde gelegt werden soll, problematisch ist. Für die Beurteilungsgrundlage in der Klinik ist die Kenntnis des Bereichs, innerhalb dessen $95 \%$ der Individuen beider Normalkollektive liegen, ausreichend. In Tabelle 2 sind neben den THE-Äquivalenten zum besseren Vergleich mit Angaben in der Literatur noch die $11 \beta$-Hydroxyätiocholanolonäquivalente aufgeführt.

Die ermittelten Normalwerte liegen im Bereich der von anderen Autoren $(3-5,7,9)$ angegebenen. Die im einzelnen vorhandenen Unterschiede dürften einerseits auf sehr kleine, uneinheitliche Kollektive, andererseits aber auch auf die Unterschiede der einzelnen Modifikationen innerhalb der Methoden, die Natriummețaperjodat verwenden, zurückzuführen sein.

Fräulein Katrin Lempart sei an dieser Stelle für ihre gewissenhafte Mitarbeit hetzlich gedankt.

Herrn Professor Dr. H. BReuer, Bonn, danken wir für die liebenswürdige Durchsicht des Manuskriptes.

\section{Literatur}

1. Appleby, J.I., G. Gibson, J. K. Normmberski und R.D. StubBs, Biochem. J. 60, 453 (1955). - 2. Wirson, H. und M. B. IIPSETr, Analytic. Biochem. 5, 217 (1963). - 3. Metcalf, M. G. J. Endocrinology 26, 415 (1963). - 4. RUtherford, E. R. und D. H. Nelson, J. Clin. Endocr., Springfield 23, 533 (1963). 5. James, V. H. T. und E. Care, J. Clin. Endocr., Springfield 24, 180 (1964). - 6. Metcalf, M. G., Clin. Chem. (New York) 12,
258 (1966). - 7. Whigham, W. R., Clin. Chem. (New York) 14, 675 (1968). - 8. GraY, C. H., D. N. Baron, R. V. Brooks und V. H. T. JAMES, Lancet, London, I/1969, 124. - 9. Few, J. D., J. Endocrinology 22, 31 (1960). - 10. LisboA, B. P., J. Chromatog. 13, 391 (1964). - 11. Pincus, G. und W. H. Pearlman, Endocrinology 29, 413 (1941). - 12. KAISER, H., Z. analyt. Chem. 209, 1 (1965).
PD. Dr. Dr. D. Stamm Abteilung für Klinische Chemie Max-Planck-Institut für Psychiatrie 8 München 23, Kraepelinstr. 10 